\title{
Harnessing the power of advocacy to improve seasonal influenza vaccination coverage in the Eastern Mediterranean Region
}

\author{
Hassan Zaraketa ${ }^{1,2}$ and Abdinasir Abubakar ${ }^{3}$
}

${ }^{1}$ Department of Experimental Pathology, Immunology \& Microbiology, Faculty of Medicine, American University of Beirut, Beirut, Lebanon. ${ }^{2}$ Center for Infectious Diseases Research, Faculty of Medicine, American University of Beirut, Beirut, Lebanon. ${ }^{3}$ Infectious Hazard Management, Department of Health Emergencies, World Health Organization, Regional Office for the Eastern Mediterranean, Cairo, Egypt. (Correspondence to: Abdinasir Abubakar: abubakara@who.int)

Citation: Zaraketa H; Abubakar A. Harnessing the power of advocacy to improve seasonal influenza vaccination coverage in the Eastern Mediterranean Region. East Mediterr Health J. 2020;26(2):138-140. https://doi.org/10.26719/2020.26.2.138

Received: 25/09/18; accepted: 26/02/19

Copyright (C) World Health Organization (WHO) 2020. Open Access. Some rights reserved. This work is available under the CC BY-NC-SA 3.0 IGO license (https://creativecommons.org/licenses/by-nc-sa/3.o/igo).

Seasonal influenza causes considerable morbidity and mortality worldwide (1). The whole population is at risk, but the threat is higher in older people, pregnant women, children, individuals with underlying comorbidities, and healthcare workers as they are susceptible to severe complications from influenza (2). Influenza vaccines are safe and effective in reducing severe outcomes of influenza infections (1). However, to be effective at the population level, high vaccination rates need to be achieved.

Many countries in the Eastern Mediterranean Region (EMR) of the World Health Organization (WHO) have implemented vaccination policies for influenza (3). However, the vaccination rates in EMRO remain very low with less than 20 doses distributed per 1000 population as compared to 45, 112, 275 doses in the Western Pacific, Europe, and Americas WHO regions, respectively (4). The exact reasons for the low vaccination rates in EMR are not well understood but are multifaceted and include misperceptions and erroneous interpretations of the efficacy and safety of the vaccine, and the threat that influenza poses to health (5-8). Additionally, the lack of regional capacity for vaccine production and so reliance on imported vaccine supplies may be a hurdle for increasing vaccine availability and distribution (4).

In this article, we describe examples of various advocacy approaches that have been shown to enhance influenza vaccine uptake. Such approaches among other initiatives and activities such as virus surveillance and burden studies can be implemented in the EMR to improve influenza awareness and vaccine uptake. Vaccine advocacy might be best characterized as the "promotion" of the best scientific knowledge, moral attitudes, and public health practices to improve immunization rates (9).

Health care workers are an important primary group to be targeted by advocacy campaigns; first, because of their risk for and role in the transmission of influenza in their practice areas and, second, because of their influence on vaccine awareness and acceptance by their patients. Influenza vaccination rates among healthcare workers in two hospitals in Qatar were doubled during the 2014/2015 season following a comprehensive campaign that comprised parallel interventions including promotional and educational tools and resources, leadership engagement, and enhanced vaccine accessibility (10). Nonetheless, simple approaches such as educational sessions and posters targeting vaccine misconceptions and improving vaccine accessibility proved useful by nearly tripling the vaccination rates among healthcare workers at a university hospital in Geneva (11). Another study found that substantial improvements in vaccination rates among nurses can be achieved by merely improving accessibility to the vaccine (12).

Another approach to improve vaccine acceptance among employees, particularly healthcare workers, is through offering gift incentives to vaccinators such as movie tickets and health books. Such incentives coupled with free and conveniently accessible vaccines and peeradvocacy resulted in substantial improvement in the vaccination rate $(>75 \%)$ among healthcare workers at a major hospital in the United States of America (USA) (13). Another hospital included influenza vaccination as one of the indicators qualifying employees for monetary bonuses which led to increased vaccination rates, from $32 \%$ in $2004 / 2005$ to $84.2 \%$ in $2005 / 2006$ (14).

Pregnant women are a priority group for vaccination, but vaccination rates among this risk group are reportedly low in the EMR (7). A randomized clinical trial revealed that brief one-on-one education of pregnant women on vaccine safety and availability as well as the potential complications from influenza substantially improved vaccine acceptance and coverage in this risk group (15). This type of advocacy and education is likely to prove effective among other risk groups.

At the level of the general population, mass media campaigns provide effective means for vaccine advocacy. A study in the USA investigated the effect of mass media coverage of influenza-related topics on influenza vaccination timing and rates among the elderly (16). Influenza-related reports in various media sources were found to positively influence influenza vaccination by shifting the timing of vaccination earlier and increasing coverage (16). Similarly, expanded media coverage of influenza illness and the vaccine was associated with improvement in vaccination rates 
among children with asthma (17).

In resource-limited settings, advocacy through social media, short message service (SMS) or emails are alternative, low-cost means of improving vaccination rates through sharing educational information or reminders for vaccination. A randomized controlled trial found that influenza vaccine coverage among highrisk patients assigned to receive an SMS vaccination reminder showed improved vaccination rates compared with the control group (18). Similar findings were reported for high-risk general practice patients in the United Kingdom and among pregnant women in New York city who received SMS vaccine reminders $(19,20)$. Phone and mail reminders effectively improved vaccination rates among people with hypertension who did not seek early seasonal influenza vaccination (21).

In conclusion, despite the good progress made in EMR in areas related to influenza vaccination, challenges remain, which highlight the need for effective communication and strong advocacy initiatives to lead the way to improve vaccine uptake and to meet public health immunization goals. Reliable information and a good understanding of the current influenza immunization practices, behaviors and policies in the Region are the mainstays of a successful advocacy campaign. Collecting regional data on influenza vaccination as well as understanding the causes of vaccine hesitancy among the target population are critical to designing evidencebased interventions. Moreover, the availability of local and regional surveillance and burden data at country and regional levels provide compelling evidence to educate the health sector and the public about influenza and the importance of vaccination. Nevertheless, every country should decide the type and number of interventions to be implemented in an advocacy campaign based on the local data and available resources.

\section{References}

1. World Health Organization. Influenza (Seasonal) [Internet]. Geneva: World Health Organization; 2018 (http://www.who.int/ news-room/fact-sheets/detail/influenza-(seasonal), accessed 28 April 2018).

2. World Health Organization. Vaccines against influenza: WHO position paper: November 2012 [Internet]. Geneva: World Health Organization; 2018 (http://www.who.int/immunization/policy/position_papers/influenza/en/, accesswed 28 March 2018).

3. Hirve S, Lambach P, Paget J, Vandemaele K, Fitzner J, Zhang W. Seasonal influenza vaccine policy, use and effectiveness in the tropics and subtropics - a systematic literature review. Influenza Other Respir Viruses. 2016;10(4):254-67.

4. Palache A, Abelin A, Hollingsworth R, Cracknell W, Jacobs C, Tsai T, et al. Survey of distribution of seasonal influenza vaccine doses in 201 countries (2004-2015): The 2003 World Health Assembly resolution on seasonal influenza vaccination coverage and the 2009 influenza pandemic have had very little impact on improving influenza control and pandemic preparedness. Vaccine. 2017 Aug 24;35(36):4681-6.

5. El Khoury G, Salameh P. Influenza Vaccination: A cross-sectional survey of knowledge, attitude and practices among the Lebanese adult population. Int J Environ Res Public Health. 2015 Dec 5;12(12):15486-97.

6. Abu-Rish EY, Elayeh ER, Mousa LA, Butanji YK, Albsoul-Younes AM. Knowledge, awareness and practices towards seasonal influenza and its vaccine: implications for future vaccination campaigns in Jordan. Fam Pract. 2016;33(6):690-7.

7. Zaraket H, Melhem N, Malik M, Khan WM, Dbaibo G, Abubakar A. Review of seasonal influenza vaccination in the Eastern Mediterranean Region: Policies, use and barriers. J Infect Public Health. 2019;12(4):472-478

8. Khowaja ZA, Soomro MI, Pirzada AK, Yoosuf MA, Kumar V. Awareness of the Pandemic H1N1 Influenza global outbreak 2009 among medical students in Karachi, Pakistan. J Infect Dev Ctries. 2011 Mar 21;5(3):151-5.

9. Balinska MA. What is vaccine advocacy?: Proposal for a definition and action. Vaccine. 2004 Mar 29;22(11):1335-42.

10. Mustafa M, Al-Khal A, Al Maslamani M, Al Soub H. Improving influenza vaccination rates of healthcare workers: a multipronged approach in Qatar. East Mediterr Health J. 2017;Jun 14;23(4):303-10.

11. Harbarth S, Siegrist CA, Schira JC, Wunderli W, Pittet D. Influenza immunization: improving compliance of healthcare workers. Infect Control Hosp Epidemiol. 1998 May;19(5):337-42.

12. Kung YM. A quality improvement project to increase influenza vaccination in healthcare personnel at a university health center. J Am Assoc Nurse Pract. 2014 Mar 1;26(3):148-54.

13. Centers for Disease Control and Prevention (CDC). Interventions to increase influenza vaccination of health-care workers-California and Minnesota. MMWR Morb Mortal Wkly Rep. 2005 Mar 4;54(8):196-9.

14. Mayoryk SA, Levy SM. Incentive Program Increases Employee Influenza Vaccine Compliance at a Chronic Hospital/Long-Term Care Facility. Am J Infect Control. 2006 Jun 1;34(5):E49.

15. Wong VWY, Fong DYT, Lok KYW, Wong JYH, Sing C, Choi AY-Y, et al. Brief education to promote maternal influenza vaccine uptake: A randomized controlled trial. Vaccine. 2016 17;34(44):5243-50.

16. Yoo B-K, Holland ML, Bhattacharya J, Phelps CE, Szilagyi PG. Effects of mass media coverage on timing and annual receipt of influenza vaccination among Medicare elderly. Health Serv Res. 2010 Oct;45(5 Pt 1):1287-309.

17. Gnanasekaran SK, Finkelstein JA, Hohman K, O’Brien M, Kruskal B, Lieu T. Parental perspectives on influenza vaccination among children with asthma. Public Health Rep Wash DC 1974. 2006 Apr;121(2):181-8.

18. Regan AK, Bloomfield L, Peters I, Effler PV. Randomized Controlled Trial of Text Message Reminders for Increasing Influenza Vaccination. Ann Fam Med. 2017 Nov;15(6):507-14. 
19. Herrett E, Williamson E, Staa T van, Ranopa M, Free C, Chadborn T, et al. Text messaging reminders for influenza vaccine in primary care: a cluster randomised controlled trial (TXT4FLUJAB). BMJ Open. 2016 Feb 1;6(2):e010069.

20. Mazzoni SE, Brewer SE, Pyrzanowski JL, Durfee MJ, Dickinson LM, Barnard JG, et al. Effect of a multi-modal intervention on immunization rates in obstetrics and gynecology clinics. Am J Obstet Gynecol. 2016;214(5):617.e1-7.

21. Minor DS, Eubanks JT, Butler KR, Wofford MR, Penman AD, Replogle WH. Improving influenza vaccination rates by targeting individuals not seeking early seasonal vaccination. Am J Med. 2010 Nov;123(11):1031-5. 